\title{
Phantom Accretion by Black Holes and the Generalized Second Law of Thermodynamics
}

\author{
J. A. S. Lima因 S. H. Pereira团 and J. E. Horvath \\ Universidade de São Paulo - Instituto de Astronomia, Geofísica e Ciências Atmosféricas \\ Rua do Matão, 1226 - 05508-090 Cidade Universitária, São Paulo, SP, Brazil \\ Daniel C. Guarientd \\ Universidade de São Paulo - Instituto de Física \\ Rua do Matão, Travessa R, \\ 187 - 05508-090 Cidade Universitária, São Paulo, SP, Brazil
}

\begin{abstract}
The accretion of a phantom fluid with non-zero chemical potential by black holes is discussed with basis on the Generalized Second Law of thermodynamics. For phantom fluids with positive temperature and negative chemical potential we demonstrate that the accretion process is possible, and that the condition guaranteeing the positiveness of the phantom fluid entropy coincides with the one required by Generalized Second Law. In particular, this result provides a complementary confirmation that cosmological phantom fluids do not need to have negative temperatures.

PACS numbers: 95.36.+x, 98.80.-k
\end{abstract}

\section{INTRODUCTION}

The discovery of the present accelerating stage of the Universe from Supernovae (SNe) type Ia observations [1] is one of the most important achievements in modern cosmology. This result has been indirectly confirmed by a large set of independent complementary observations, involving the temperature anisotropies of the cosmic microwave background [2], the X-ray surface brightness from galaxy clusters [3] and the age of the universe from globular clusters and old high redshift galaxies [4]. In the context of general relativity, an accelerating stage can be explained by assuming the existence of an exotic substance with negative pressure dubbed dark energy (or quintessence) which represents an intriguing evidence for physics beyond the domain of the standard model of particle physics.

Theoretically, there are many candidates to represent this extra non-luminous (in addition to cold dark matter) relativistic component [5]. Many authors have worked on the idea that the unknown, uncoupled dark energy component is due exclusively to a minimally coupled scalar field (quintessence field) which has not yet reached its ground state and whose current dynamics is basically determined by its potential energy [6]. A special attention has also been paid to the so-called XCDM cosmologies which are phenomenologically described by an equation of state (EoS) of the form [7]

$$
p=\omega \rho,
$$

where $p$ and $\rho$ denotes the pressure and energy density, respectively, and $\omega$ is a constant negative parameter. The case $\omega=-1$ corresponds to a positive cosmological constant, or

\footnotetext{
*Electronic address: limajas@ astro.iag.usp.br

†Electronic address: spereira@astro.iag.usp.br

‡Electronic address: foton@astro.iag.usp.br

${ }^{\S}$ Electronic address: carrasco@fma.if.usp.br
}

vacuum energy, while for $\omega<-1$ we have the so called phantom dark energy regime or phantom fluids. Depending on the form of potential, this kind of fluid can also be represented by a scalar field model [8].

It is widely known that $\mathrm{SNe}$ data alone rules out nonaccelerating models with very high significance level, and that even dark energy candidates with $\omega>-2 / 3$ are strongly disfavored. Actually, some analyzes based on the combination of $\mathrm{SNe}$ and large scale structure data imply that the EoS parameter lies within the range $-1.48<\omega<-0.72$ at $95 \%$ confidence level [9]. Therefore, the possibility of a phantom regime $(\omega<-1)$ cannot be excluded.

The study of the phantom regime present some peculiar features, for example, stability issues [10, 11], increasing energy density with cosmic time, the possibility of superluminal sound speed [12] and even the violation of the dominant energy condition is possible (as the phantom fluid must satisfy $\rho+p<0$ ). The dominance of the cosmological content by a phantom fluid implies that the universe will undergo a very fast expansion in the future when it finally reaches the big-rip singularity.

Another intriguing point is related to the thermodynamic properties underlying the phantom energy fluids. Some earlier studies developed by Lima and coworkers [13, 14] shown that the temperature of the phantom fluid without chemical potential is positive definite but its co-moving entropy, $S_{\mathrm{ph}}<0$, is always negative (for a different analysis showing the same result see the paper by Brevik et. al. [15]). Therefore, unless one disregards the statistical microscopic concept of entropy from which $S>0$, the phantom fluid cannot exist in nature (see also [16] for a different approach based on the idea that the temperature of phantom fields is negative).

Later on, Izquierdo and Pavón [17] discussed the phantom energy in the context of the generalized second law (GSL) of thermodynamics. It was found that although the entropy constraint be violated, the GSL may be satisfied, that is, $\dot{S}=$ $\dot{S}_{\mathrm{ph}}+\dot{S}_{\mathrm{h}} \geq 0$, where $S_{\mathrm{h}}$ is the horizon entropy.

More recently, the thermodynamic and statistical properties of phantom fluids were reexamined by considering the exis- 
tence of a non-zero chemical potential $(\mu)$. In this case, it was found that the entropy condition, $S \geq 0$, implies that the possible values of $\omega$ are heavily dependent on the value, as well as on the sign of the chemical potential [18] (for a related study in K-essence dark energy models see the paper by Bilić [19]). For $\mu>0$, the $\omega$-parameter must be strictly greater than -1 (vacuum is forbidden) while for $\mu<0$ not only the vacuum but even a phantom-like behavior $(\omega<-1)$ is allowed. In any case, the ratio between the chemical potential and temperature remains constant, that is, $\mu / T=\mu_{0} / T_{0}$, where $\mu_{0}, T_{0}$ are the present day values of the chemical potential and temperature, respectively. Still more important, the positiveness of the entropy is preserved for all kinds of dark energy components as long as the condition

$$
\omega \geq-1+\frac{\mu_{0} n_{0}}{\rho_{0}}
$$

is satisfied, where $n_{0}$ and $\rho_{0}$ are, respectively, the present values of the particle number density and energy density of the dark energy fluid described by Eq. (1). Actually, if the chemical potential is negative, the above expression shows that the $\omega$ parameter is effectively describing a phantom fluid.

In this context, it is interesting to investigate the constraints associated to the validity of GSL and the possibility of a positive critical mass enabling the phantom accretion onto black holes. As we shall see, the positiveness of this mass leads to a remarkable result, namely: the same condition for the $\omega$ parameter given above is also required by GSL. In particular, this means that cosmological phantom fluids do not need to have negative temperatures. The present study is complemented with some numerical estimates of the critical mass based on the Holographic Bound, that is, the idea that there is an upper limit to the entropy inside the "surface" limiting the system.

\section{ACCRETION PROCESS OF PHANTOM FLUIDS}

Following the results presented in [18], let us now discuss the thermodynamic aspects governing the accretion of of phantom fluids by black holes when the chemical potential of the phantom fluid is non zero. The main results obtained here will be compared with the recent analysis by Pacheco and Horvath [20] in the absence of chemical potential.

The change on the black hole mass due to the accretion process in a universe filled with a dark energy fluid was first investigated by Babichev, Dokuchaev and Eroshenko [21]. In the case of phantom fluids, they found that the black hole mass changes at a negative rate, that is, $\dot{M}<0$. Mathematically, such a behavior can be readily understood. In a general accreting process the black hole mass rate is proportional to the enthalpy density of the fluid $(\rho+p)$. Therefore, when the dominant energy condition (DEC) is obeyed (violated), the black hole mass will increase (decrease) during the accretion process. In this second case, the immediate consequence is that the area of the black hole horizon will decrease along with its entropy $\left(S \propto M^{2}\right.$, where $\mathrm{M}$ is the mass of the Schwarzschild black hole).

On the other hand, Gao and collaborators [22] claimed that the back-reaction provoked by the accretion of phantom matter on the black hole metric was ignored in the mentioned calculations. In a low matter density background, this effect can be safely ignored, but if the background matter density is large (comparable to the black hole density), the metric describing this black hole will be significantly modified, and the backreaction must necessarily be considered. Different from the authors of [21] they found that the physical black hole mass will not decrease but rather grows due to the accretion of phantom fluid, so that $\dot{M}>0$. However, Martín-Moruno et al. [23] have argued that the Gao et al. findings are misleading because it was assumed that the black hole mass function is proportional to the scale factor. In particular, the entropy of the fluid has not been taken into account in the solution for the accretion process, and, as such, the result seems to be based on a somewhat incomplete picture. For completeness and by considering that the results are still under debate, in what follows we will discuss the equilibrium conditions for both cases $(\dot{M}>0$ and $\dot{M}<0)$.

\section{A. Phantom Accretion by Black Holes: GSL Constraints}

In terms of the present day quantities, the energy density of a dark fluid can be written as [13, 14, 15]

$$
\rho=\rho_{0}\left(\frac{T}{T_{0}}\right)^{\frac{1+\omega}{\omega}}
$$

whereas its entropy (including a chemical potential) reads [18]

$$
S=\left[\frac{(1+\omega) \rho_{0}-\mu_{0} n_{0}}{T_{0}}\right]\left(\frac{T}{T_{0}}\right)^{\frac{1}{\omega}} .
$$

where $T$ is the temperature ( $T_{0}$ is its present day value). It thus follows that the total entropy of the system consisting of a (Schwarzschild) black hole plus a dark energy fluid reads (unless explicitly stated, in our units $\hbar=k_{B}=c=1$ ),

$$
S=4 \pi G M^{2}+\left[\frac{(1+\omega) \rho_{0}-\mu_{0} n_{0}}{T_{0}}\right]\left(\frac{\rho}{\rho_{0}}\right)^{\frac{1}{1+\omega}} V,
$$

where the first term represents the black hole entropy and the second term is the phantom fluid entropy inside a co-moving volume $V$, written in terms of the energy density. Now, due to the accretion process, in an arbitrarily short time interval, the black hole mass varies by $\Delta M$ and the phantom field energy varies by $\Delta \rho$. Therefore, the total entropy variation within the cavity takes the form 


$$
\Delta S=8 \pi G M \Delta M+\frac{1}{(1+\omega)}\left[\frac{(1+\omega) \rho_{0}-\mu_{0} n_{0}}{T_{0} \rho_{0}}\right]\left(\frac{\rho}{\rho_{0}}\right)^{-\frac{\omega}{1+\omega}} V \Delta \rho .
$$

For a phantom fluid modelled by a scalar field, only the kinetic

term contributes to the accretion so that the energy conservation inside the cavity implies that

$$
\Delta M=-\frac{1}{2}(1+\omega) V \Delta \rho
$$

Now, by inserting (7) into (6), we obtain an expression for the total entropy variation of the black hole plus dark energy:

$$
\Delta S=\left\{8 \pi G M-\frac{2}{(1+\omega)^{2}}\left[\frac{(1+\omega) \rho_{0}-\mu_{0} n_{0}}{T_{0} \rho_{0}}\right]\left(\frac{\rho}{\rho_{0}}\right)^{-\frac{\omega}{1+\omega}}\right\} \Delta M .
$$

We move now to analyze the two cases previously presented. According to Gao et al. [22], the mass of the black hole increases with time during the phantom accretion process, that is, $\Delta M \geq 0$. In order to satisfy the GSL $(\Delta S \geq 0)$, we must have the term inside the curly brackets appearing in (8) positive definite thereby yielding the condition:

$M \geq M_{\text {crit }}=\frac{1}{4 \pi G(1+\omega)^{2}}\left[(1+\omega)-\frac{\mu_{0} n_{0}}{\rho_{0}}\right] \frac{1}{T_{0}}\left(\frac{\rho}{\rho_{0}}\right)^{-\frac{\omega}{1+\omega}}$.

Note that for $\mu_{0}=0$ the above critical mass reduces to the one derived in Ref. [20] (see their equation (14)). In this case, the critical mass is negative when $\omega<-1$ and the inevitable conclusion is that the process is physically forbidden. In the point of view of Refs. [14, 15], such a result should be physically expected since phantom fluids with negative temperature cannot exist in nature. On the other hand, we see that for negative values of $\mu_{0}$ there exists a positive critical mass above which the black hole can accrete the phantom fluid.

If we adopt the results from Babichev et al. [21], where $\Delta M<0$, the condition to the mass is $M \leq M_{\text {crit }}$. Therefore, only black holes with mass below the critical mass can accrete phantom energy.

An interesting result emerging from the above discussion based on the validity of GSL is that to keep the positiveness of the critical mass, the following condition must be satisfied (the term outside the square brackets in (9) is positive definite and thus the term inside the square brackets must be positive as well)

$$
\omega \geq-1+\frac{\mu_{0} n_{0}}{\rho_{0}},
$$

which is just condition (2) previously obtained in [18] from a thermodynamical analysis based exclusively on the phantom fluid thermodynamic properties. The fact that the same condition emerges naturally from the GSL constraints reinforces the claims that phantom fluids must have positive temperatures.

\section{B. Critical Mass Estimates}

The above constraint has an additional interesting property. It can be used to give an upper bound estimate to the present day value of the chemical potential, more precisely to the product $\mu_{0} n_{0}$. Notice that the inequality (2) can be written as

$$
\mu_{0} \leq \frac{\rho_{0}}{n_{0}}(1+\omega) .
$$

It should also be recalled that the condition (2) for the chemical potential is a general result, and, as such, it is not related to our previous analysis of phantom fluid accretion by black holes with basis on GSL. Since the chemical potential has the dimension of energy per particle, the product $\mu_{0} n_{0}$ has the dimension of energy density, like $\rho_{0}$. Assuming that our universe is flat, the critical density is given by $\rho_{c} \equiv 3 H_{0}^{2} / 8 \pi G \simeq 4 \times 10^{-47} \mathrm{GeV}^{4}$. If the dark energy contributes with about $70 \%$ of the matter content of the universe, we may suppose that the present day value of the dark energy density is given by $\rho_{0} \simeq 0.7 \times \rho_{c} \simeq 2.8 \times 10^{-47} \mathrm{GeV}^{4}$. This allows us to estimate

$$
\mu_{0} n_{0} \leq(1+\omega) \rho_{c} .
$$

Taking as an example the value $\omega=-3 / 2$, we have $\mu_{0} n_{0} \leq-1.4 \times 10^{-47} \mathrm{GeV}^{4}$.

In order to study some limits of (9), let us define $\alpha \equiv-\mu_{0} n_{0} / \rho_{0}$ the ratio between the chemical potential density 
and the energy density, which is a constant dependent only on present day values. Using the relation (3), we rewrite the critical mass (9) as

$$
M_{\text {crit }}=4.5 \times 10^{-20}\left[\alpha-\frac{1}{2}\right] \frac{M_{\odot}}{T_{G e V}},
$$

where $M_{\odot}$ is the solar mass, $T$ is the temperature of the phantom fluid, given in $\mathrm{GeV}$, and $\omega=-3 / 2$ is used. This shows that the critical mass is proportional to the inverse of the temperature of the phantom fluid and also depends on $\alpha$.

\section{CRITICAL MASS FROM HOLOGRAPHIC PRINCIPLE}

A more quantitative analysis of the critical mass value requires knowledge of its entropy, and consequently of its chemical potential, which remains indeterminate since the remaining constants $n_{0}, \rho_{0}$ and $T_{0}$ are unknown. However, an upper bound can be derived from Holographic Principle bound which states that

$$
\frac{4 \pi}{3} R^{3} s \leq \frac{\pi}{l_{P}^{2}} R^{2}
$$

where $R$ is the event horizon radius, $s$ is the entropy density and $l_{P}$ is the Planck length. It can be showed that, in a phantom dominated universe, the Holographic Bound is satisfied if (see [20] and references therein)

$$
s_{0} \leq \frac{3|1+3 \omega|}{8} \frac{\sqrt{\Omega_{D E}} H_{0}}{l_{P}^{2}} \simeq 7.2 \times 10^{-5}|1+3 \omega| \mathrm{GeV}^{-3}
$$

In our case, using (4), we have

$$
-\mu_{0} n_{0} \leq-(1+\omega) \rho_{0}+T_{0} \frac{3|1+3 \omega|}{8} \frac{\sqrt{\Omega_{D E}} H_{0}}{l_{P}^{2}},
$$

or in terms of the $\alpha$ parameter defined above

$$
\alpha \leq \frac{1}{2}+3.9 \times 10^{42} T_{0}
$$

where we have used $\omega=-3 / 2$ and $\rho_{0}=0.7 \times \rho_{\text {crit }}$, and $T_{0}$ is given in $\mathrm{GeV}$.

Now, by considering the case of very small temperature, $\alpha \approx 1 / 2$ if we take $T_{0} \simeq 0$, and thus $M_{\text {crit }} \simeq 0$. This shows that all black holes can accrete in the case $\Delta M \geq 0$, but the process is forbidden for case $\Delta M \leq 0$.

As discussed by several authors, the thermodynamic treatment of dark energy fluids implies that its temperature increases in the course of the expansion. Thus, a more interesting case occurs if we take, for instance, $T_{0} \simeq 10^{-19} \mathrm{GeV}$ $\simeq 10^{-6} \mathrm{~K}$, and so $\alpha \approx 10^{24}$. We have the critical mass $M_{\text {crit }} \simeq 4.6 \times 10^{3} M_{\odot} / T$. Today this critical mass is $M_{\text {crit }} \simeq 4.6 \times$

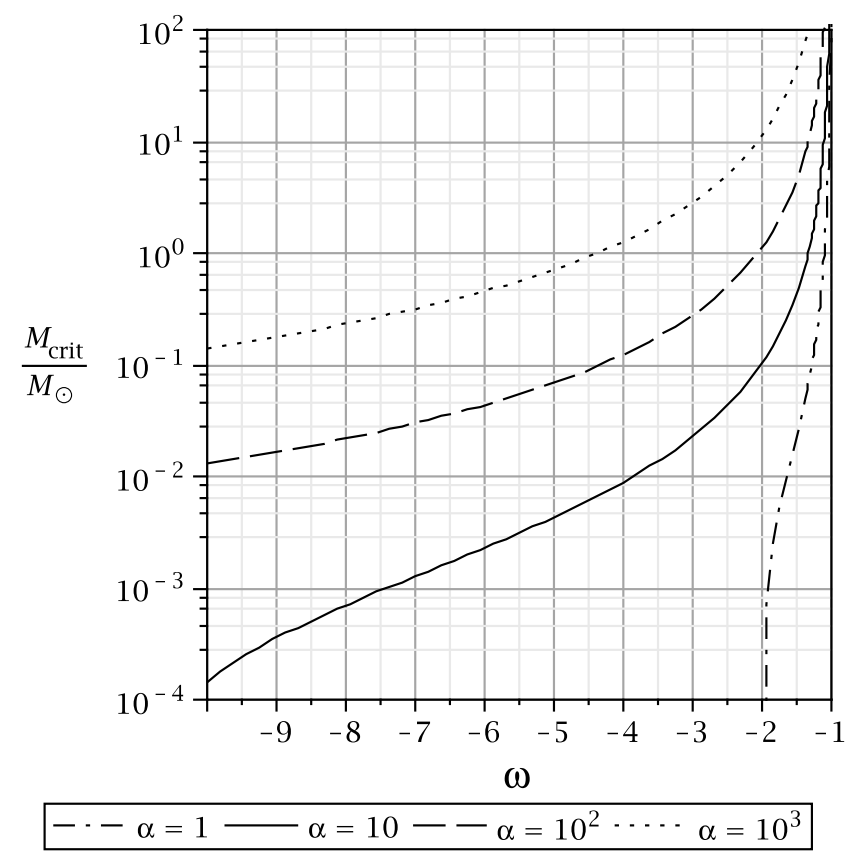

FIG. 1: The present Black hole critical mass as a function of $\omega$ for some selected values of $\alpha$.

$10^{22} M_{\odot}$, a very large mass, but of the same order of that obtained in [20] by a different method.

A more realistic case occur if we take $\alpha \approx 10$. In this case the critical mass today is $M_{\text {crit }} \simeq 2.2 M_{\odot}$, the order of a solar mass. It is interesting to note the dependence of the critical mass with $\omega$ for some values of $\alpha$. It is plotted in Fig. 1.

\section{CONCLUSIONS}

In this work we have discussed the accretion of phantom fluids with negative chemical potential by black holes. As we have seen, there is a positive critical mass in order to enable the phantom accretion. Some numerical estimates constraining the critical mass threshold based on the adoption of reasonable values of the chemical potential, or more directly, in terms of the Holographic Bound, have also be presented, even if the latter has not been rigourously proved to hold for arbitrary space-times.

As physically expected, the Generalized Second Law of thermodynamics determines the thermodynamic viability of the whole process and the amount of dark energy accretion. Depending on the kind of analysis adopted, the process may either increase or decrease the mass range of black holes accreting the phantom fluid along the history of the universe. More interesting, the positiveness of the critical mass (based on GSL) leads to the same constraint involving the present day values of the physical quantities specifying the phantom fluid and previously obtained through a thermodynamic analysis of the phantom energy alone [18]. In other words, phantom fluids with zero chemical potential are not consistent because 
they require either a negative entropy (which is microscopically unacceptable) or a negative temperature (which needs a bounded spectrum which has not been justified from any scalar field model).

\section{Acknowledgments}

The authors would like to thank V. C. Busti, J. V. Cunha, J. F. Jesus, A. C. Guimarães, R. Holanda, R. C. Santos and P.
I. Braun for helpful discussions. JASL is partially supported by CNPq and FAPESP No. 04/13668-0 and SHP is supported by CNPq No. 150920/2007-5 (Brazilian Research Agencies). DCG and JEH are supported by CNPq through grants and fellowships.
[1] A. G. Riess et al., Astron. J. 116, 1009 (1998); S. Perlmutter et al., Astrophys. J. 517, 565 (1999); P. Astier et al., Astron. Astrophys. 447, 31 (2006); A. G. Riess et al., Astrophys. J. 659, 98 (2007).

[2] P. de Bernardis, et al., Nature 404, 955 (2000); D. N. Spergel, et al., Astrophys. J. Suppl. Ser. 148, 175 (2003); D. N. Spergel et al., Astrophys. J. Suppl. Ser. 170, 377 (2007).

[3] J. A. S. Lima, J. V. Cunha and J. S. Alcaniz, Phys. Rev. D 68, 023510 (2003), |astro-ph/0303388|; S. W. Allen et al., [arXiv:0706.0033].

[4] J. S. Dunlop et al., Nature 381, 581 (1996); L. Krauss, Astrophys. J. 480, 466 (1997); J. S. Alcaniz and J. A. S. Lima, Astrophys. J. 521, L87 (1999), [astro-ph/9902298]; Astrophys. J. 550, L133 (2001), [astro-ph/0101544]; J. A. S. Lima and J. S. Alcaniz, Mon. Not. Roy. Astron. Soc. 317893 (2000), [astro-ph/0005441]; J. V. Cunha and R. C. Santos, Int. J. Mod. Phys. D13, 1321 (2004), astro-ph/0402169]; D. Jain and A. Dev, Phys. Lett. B633, 436 (2006), [astro-ph/0509212]; J. F. Jesus, Gen. Rel. Grav. 40, 791 (2008).

[5] T. Padmanabhan, Phys. Rept. 380, 235 (2003); P. J. E. Peebles and B. Ratra, Rev. Mod. Phys. 75, 559 (2003); J. A. S. Lima, Braz. Jour. Phys. 34, 194 (2004), |astro-ph/0402109|; V. Sahni and A. Starobinsky, Int. J. Mod. Phys. D15, 2105 (2006).

[6] P. J. E. Peebles, Astrophys. J. Lett. 325, L17 (1988); B. Ratra and P. J. E. Peebles, Phys. Rev. D 37, 3406 (1988); R. R. Caldwell, R. Dave, P. J. Steinhardt, Phys. Rev. Lett. 80, 1582 (1998); C. Wetterich, Nucl. Phys. B302, 668 (1998); R. Caldwell, R. Dave and P. Steinhardt, Phys. Rev. Lett. D 59, 123504 (1999); R. Caldwell, Braz. J. Phys. 30, 215 (2000); P. G. Ferreira and M. Joyce, Phys. Rev. D 58, 023503 (1998); A. Albrecht and C. Skordis, Phys. Rev. Lett. 84, 2076 (2000); C. Rubano et al., Phys. Rev. D 69, 103510 (2004); V. Faraoni and M. N. Jensen, Class. Quant. Grav. 23, 3005 (2006); F. C. Carvalho et al., Phys. Rev. Lett. 97, 081301 (2006), [astro-ph/0608439]; J. E. Carrillo, J. M. Silva and J. A. S. Lima, |arXiv:0806.3299.

[7] M. S. Turner and M. White, Phys. Rev. D 56, R4439 (1997); T. Chiba, N. Sugiyama and T. Nakamura, Mon. Not. Roy. Ast. Soc. 289, L5 (1997); J. A. S. Lima and J. S. Alcaniz, Astron. Astrophys, 357393 (2000), astro-ph/0003189]; Astrophys. J. 566, 15 (2002), [astro-ph/0109047]; J. F. Jesus et al., [arXiv:0806.1366].
[8] R. R. Caldwell, M. Kamionkowsky and N. N. Weinberg, Phys. Rev. Lett. 91071301 (2003); S. M. Carroll, M. Hoffman, M. Trodden, Phys. Rev. D 68, 023509 (2003); P. F. Gonzalez-Diaz, Phys. Rev. D 68, 021303 (2003); S. Nojiri, S.D. Odintsov, Phys. Lett. B562, 147 (2003); B565, 1 (2003); Y.-S. Piao, E. Zhou, Phys. Rev. D 68 (2003) 083515; J. S. Alcaniz, Phys. Rev. D 69 (2004) 083521, [astro-ph/ 0312424]; S. Hannestad, E. Mortsell, Phys. Rev. D 66 (2002) 063508; T. R. Choudhury, T. Padmanabhan, [astro-ph/0311622]; R. C. Santos and J. A. S. Lima, Phys. Rev. D 77, 083505 (2008), arXiv:0803.1865|.

[9] J. L. Tonry et. al., Astrophys. J. 594, 1 (2003).

[10] P. H. Frampton, [hep-th/0302007].

[11] J. M. Cline, S. Y. Jeon and G. D. Moore, Phys. Rev. D 70, 043543 (2004).

[12] A. E. Schulz, M. J. White, Phys. Rev. D 64, 043514 (2001); J. G. Hao and X. Z. Li, Phys. Rev. D 67, 107303 (2003).

[13] J. A. S. Lima and A. Maia Jr., Phys. Rev. D 52, 56 (1995), |gr-qc/9505052]; Int. J. Theor. Phys, 34, 9 (1995), |gr-qc/9505052|; J. A. S. Lima and J. Santos, Int. J. Theor. Phys. 34, 143 (1995); J. A. E. Carrillo, J. A. S. Lima and A. Maia Jr., Int. J. Theor. Phys. 35, 2013 (1996), |hep-th/9906016|.

[14] J. A. S. Lima and J. S. Alcaniz, Phys. Lett. B600, 191 (2004), astro-ph/0402265].

[15] I. Brevik, S. Nojiri, S. D. Odintsov and L. Vanzo, Phys. Rev. D 70, 043520 (2004).

[16] P. F. González-Díaz and C. L. Sigüenza, Nucl. Phys. B697, 363 (2004); Phys. Lett.B589, 78 (2004).

[17] G. Izquierdo and D. Pavón, Phys. Lett. B633, 420 (2006); Phys. Lett. B639, 1 (2006), |gr-qc/0612092].

[18] J. A. S. Lima and S. H. Pereira, |arXiv:0801.0323|; S. H. Pereira and J. A. S. Lima, arXiv:0806.0682]; S. H. Pereira, arXiv:0806.3701.

[19] N. Bilić, arXiv:0806.0642].

[20] J. A. Freitas Pacheco and J. E. Horvath, Class. Quant. Grav. 24, 5427 (2007).

[21] E. Babichev, V. Dokuchaev and Yu. Eroshenko, Phys. Rev. Lett. 93 (2004) 021102.

[22] C. Gao, X. Chen, V. Faraoni and Y. Shen, arXiv:0802.1298].

[23] P. Martín-Moruno, A. L. Marrakchi, S. Robles-Pérez and P. F. González-Díaz, |arXiv:0803.2005]. 